\title{
ESTRUTURA E DINÂMICA DA COMUNIDADE FITOPLANCTÔNICA DOS RESERVATÓRIOS BILLINGS E GUARAPIRANGA, SP, BRASIL
}

Tese apresentada ao Instituto de Ciências Biomédicas da Universidade de São Paulo, para obtenção do Título de Doutor em Ciências.

Área de concentração: Microbiologia

Orientador: Prof. Dr. José Luiz Negrão Mucci 


\section{RESUMO}

GEMELGO, M. C. P. Estrutura e dinâmica da comunidade fitoplanctônica dos Reservatórios Billings e Guarapiranga, SP, Brasil. 161 f. Tese (Doutorado em Ciências) - Instituto de Ciências Biomédicas, Universidade de São Paulo, São Paulo, 2008.

Atualmente, a formação de grandes aglomerados urbanos e industriais, com crescente necessidade de água para abastecimento, além de irrigação e lazer, faz com que as atividades humanas sejam cada vez mais dependentes da disponibilidade das águas continentais. Os Reservatórios Billings e Guarapiranga integram uma das principais fontes de abastecimento público da cidade de São Paulo, suprindo água para milhões de pessoas. Esses reservatórios estão sofrendo intensa ação antrópica devido a despejos domésticos, industriais e agropecuários. O aporte de nutrientes em corpos hídricos, advindos de efluentes domésticos e industriais, fertilizantes agrícolas e de outras fontes, leva à eutrofização das águas propiciando, assim, condições ideais para a proliferação do fitoplâncton. Dentre esses organismos destacam-se as Cyanobacteria que atraem a atenção não só de pesquisadores e técnicos que controlam a qualidade das águas, mas também do público em geral por sua resposta imediata a eutrofização de reservatórios, sob forma de florações. Com base no exposto, foi levantada a hipótese de que existiam diferenças quantitativas e qualitativas entre as comunidades fitoplanctônicas nos reservatórios, condicionadas por fatores ambientais. Assim, os objetivos do trabalho foram acompanhar a dinâmica da comunidade fitoplanctônica durante um ciclo sazonal e verificar a influência das variáveis físicas e químicas da água sobre a estrutura dessa comunidade. As amostras mensais, realizadas na superfície da coluna d'água, foram analisadas ao microscópio óptico binocular e a análise quantitativa foi feita ao microscópio invertido. A maior densidade (ind. $\mathrm{mL}^{-1}$ ) da comunidade fitoplanctônica em ambos os reservatórios, foi representada por organismos das Classes Chlorophyceae e Cyanobacteria, sendo a última dominante no Reservatório Billings e abundante no Reservatório Guarapiranga, nas estações seca e chuvosa. Os principais fatores ambientais que interferiram na dinâmica da comunidade fitoplanctônica, em ambos os reservatórios, foram elevados valores de temperatura da água, de $\mathrm{pH}$ e de nutrientes.

Palavras-chave: Fitoplâncton. Chlorophyceae. Cyanobacteria. Reservatórios de abastecimento. 


\section{ABSTRACT}

GEMELGO, M. C. P. Billings and Guarapiranga reservoirs (SP, Brazil): phytoplankton community structure and dynamics. 161 p. Doctorate Thesis (Sciences) - Instituto de Ciências Biomédicas, Universidade de São Paulo, São Paulo, 2008.

In present days, the development of huge urban and industrial agglomerations, that have growing need of water supply, irrigation and leisure, make human activities more and more dependent of freshwater availability. Billings and Guarapiranga reservoirs are part of the main public water supply of São Paulo city, supplying water to millions of people. These reservoirs undergo intense antropic action due to the disposal of untreated domestic, industrial and agricultural wastes. The nutrient input to hidric resources from domestic and industrial effluents, fertilizers and other sources, causes eutrophication, thus creating ideal conditions to phytoplankton blooms to occur. Among these organisms are the Cyanobacteria, which are of concern not only to researchers and technicians responsible for the water quality, but also to the population in general, due to their immediate response to water eutrophication, causing blooms. Therefore, it was supposed that there are qualitative and quantitative differences between the phytoplankton communities on both reservoirs, caused by environmental factors. Thus, the objectives of the present work were to follow up the phytoplankton community along one seasonal cycle and to identify the influence of physical and chemical parameters of the water on the community structure. Monthly samples were taken from surface water and analyzed under a binocular optical microscope and the quantitative analyses were performed under an inverted microscope. The greatest phytoplankton density (ind. $\mathrm{mL}^{-1}$ ) in both reservoirs was due to individuals of Chlorophyceae and Cyanobacteria classes, the last being dominant in Billings reservoir and abundant in Guarapiranga reservoir, be it rainy or dry season. The main environmental factors that interferred in phytoplankton dynamics were high temperature values, $\mathrm{pH}$ and nutrients, in both reservoirs.

Key words: Phytoplankton. Chlorophyceae. Cyanobacteria. Water supply reservoirs. 


\section{INTRODUÇÃO}

Reservatórios artificiais ocupam cerca de $7.500 \mathrm{Km}^{2}$ de águas represadas em todo o planeta. O gerenciamento desses ecossistemas artificiais, da qualidade de sua água e das bacias hidrográficas na qual se inserem esses reservatórios é de fundamental importância para o desenvolvimento sustentável (STRAŜKRABA e TUNDISI, 2000).

Os reservatórios vêm sofrendo, no mundo todo, os impactos provocados pela proliferação das atividades antrópicas nos seus entornos. No Brasil, a deterioração dos rios, lagos, represas e outros mananciais, compromete a qualidade da água, a manutenção dos hábitats aquáticos, a biodiversidade e a saúde humana (GENTIL, 2000).

Atualmente, a formação de grandes aglomerados urbanos e industriais, com crescente necessidade de água para abastecimento doméstico e industrial, além de irrigação e lazer, faz com que a quase totalidade das atividades humanas seja cada vez mais dependente da disponibilidade das águas continentais. A dependência do homem moderno, em relação aos ecossistemas aquáticos, é ainda mais evidente nas regiões altamente industrializadas na qual a demanda de água "per capita" tem se tornado cada vez maior. Além disso, nessas regiões, grande parte dos efluentes domésticos e industriais é lançada diretamente nos corpos d'água, reduzindo ainda mais a possibilidade de utilização dos recursos hídricos (ESTEVES, 1998; TUNDISI, 2003).

O panorama econômico paulista ganha destaque com a agricultura e a industrialização. A crescente demanda por alimentos expandiu a atividade agrícola no estado, sendo responsável pelo grande impacto sobre os recursos hídricos por meio do uso de grandes quantidades de fertilizantes químicos, contaminando o solo e as águas. Por outro lado, a indústria serviu-se da água utilizando-a como matéria prima, como parte de seus processos industriais e também para diluir e lançar ao ambiente os seus efluentes carregados de metais pesados e compostos tóxicos (MATSUZAKI, 2002).

O problema da escassez de água está relacionado à distribuição desigual do recurso nas regiões. Além disso, o desenvolvimento acelerado de novas tecnologias e os novos processos produtivos, aliados às aglomerações de populações nos grandes centros urbanos e industriais, acarreta, ao longo dos anos, a degradação da qualidade da água com prejuízos à saúde pública, economia e ao meio ambiente (MATSUZAKI, 2007). 
A Unidade de Gerenciamento de Recursos Hídricos (UGRHI) da Bacia do Alto Tiête é composta por 34 municípios e abrange a parte superior do Rio Tietê, desde a sua cabeceira até a barragem do Reservatório de Pirapora, numa extensão de $133 \mathrm{~km}$. Abriga quase a metade da população do Estado e compreende, em seu território, parte da Região Metropolitana de São Paulo (RMSP) (CETESB, 2007).

De acordo com estudo elaborado pela Fundação de Apoio à Universidade de São Paulo (Fusp) para o Comitê da Bacia Hidrográfica do Alto Tietê, no ano de 2000, a RMSP contabilizava 17,8 milhões de habitantes em seus 39 municípios, respondendo por $18 \%$ do Produto Interno Bruto do país - maior do que o de países como Chile e Irlanda. Esta concentração populacional, entretanto, tem causado um "stress hídrico" na região, já que o sistema de abastecimento de água trabalha sem nenhuma folga. Ou seja, a oferta e a demanda são praticamente as mesmas (INSTITUTO DE ENGENHARIA, 2003).

Estudos mostram ainda que as taxas de crescimento populacional da RMSP baixam a cada ano, porém de maneira não uniforme. Mesmo assim as projeções indicam que a região terá cerca de 20 milhões de habitantes em 2010 e entre 22 e 24 milhões em 2020. Esta equação (crescimento da população + baixíssima disponibilidade hídrica) faz com que o problema de abastecimento de água da região ganhe contornos ainda mais preocupantes. A situação se agrava quando a distribuição desigual desta população faz com que áreas de proteção sejam ocupadas, aumentando os riscos de contaminação de mananciais. Assim, não basta que o Poder Público disponibilize mais água para o abastecimento. É preciso garantir a proteção aos mananciais já existentes - deixando claro que ações corretivas são sempre mais custosas do que as preventivas (INSTITUTO DE ENGENHARIA, 2003).

O problema é notadamente grave nos Reservatórios Billings e Guarapiranga, que sofrem com o processo de eutrofização (enriquecimento por nutrientes, principalmente fosfatos e compostos nitrogenados) da água, agredidos pelo crescimento populacional nas áreas de proteção (INSTITUTO DE ENGENHARIA, 2003).

Além disso, devido à escassez de água no Reservatório Guarapiranga, que abastece milhões de pessoas na cidade de São Paulo, foi necessária a construção de um canal interligando o Reservatório Billings (braço Taquacetuba) ao Reservatório Guarapiranga, a fim de resolver problemas de déficit hídrico nesse reservatório, cuja operação de bombeamento de água teve início em agosto de 2000 (BEYRUTH, 2000; CAPOBIANCO e WHATELY, 2002; CARVALHO, 2003; MATSUZAKI, 2007; TUNDISI, 2003).

A evolução do uso do solo da região tem se caracterizado pela substituição da vegetação natural e da pequena atividade agrícola por um processo de intensa urbanização. 
Apesar das condições físicas desfavoráveis (relevos acidentados e várzeas), que acabam ocasionando problemas como escorregamento, erosão, assoreamento e da existência das Leis de Proteção aos Mananciais, a ocupação da área vem se acelerando, sobretudo nos últimos anos. Atualmente, a região caracteriza-se por grandes contrastes, associando chácaras de recreio com favelas e loteamentos irregulares e clandestinos (CARVALHO, 2003; PRESOTTO e CABIANCA, 2006).

Segundo Straškraba ${ }^{1}$ et al. (1993) apud Calijuri (1999), a complexidade em manejar a qualidade da água de um reservatório reside na natureza dinâmica desse sistema, na interferência do homem e na viabilidade de implantação de ações de monitoramento e gerenciamento dos sistemas aquáticos.

O desenvolvimento de programas eficientes de manejo requer conhecimento da dinâmica e metabolismo do sistema ecológico. Compreender este metabolismo significa estudar os processos envolvidos e, consequentemente, as transferências de energia dos seus diversos níveis tróficos. A base para o conhecimento do fluxo de energia de um sistema é a sua produção primária (CALIJURI, 1999).

Ainda segundo Calijuri (1999), a produção da matéria fotossintética, pela comunidade fitoplanctônica, é o processo mais importante do metabolismo de um sistema. Pela fotossíntese, a energia luminosa é convertida em energia química, estocável em compostos orgânicos como carboidratos, lipídeos e proteínas. A quantidade de matéria orgânica nova produzida pelos autótrofos é chamada de produção primária.

A produção primária fitoplanctônica é controlada pela interação de muitos fatores que, segundo Findenegg ${ }^{2}$ (1974) apud Calijuri (1999), são divididos em três grupos:

1. fatores físicos - originados diretamente da radiação solar, assim como condições de luz e temperatura;

2. fatores químicos - conteúdo de nutrientes na zona eufótica;

3. fatores bióticos - interações dos organismos presentes na comunidade fitoplanctônica que podem favorecer ou dificultar a produção de certas espécies.

Estes fatores estão mutuamente relacionados e determinam a distribuição dos organismos no espaço e tempo.

\footnotetext{
${ }^{1}$ STRAŠKRABA, M. et al. Framework for investigation and evaluation of reservoir water quality in Czechoslovakia. In: STRAŠKRABA, M; TUNDISI, J. G.; DUCAN, A. (Ed.). Comparative reservoir Limnology and Water Quality Management. Netherlands: Kluwer Academic Plublishers, 1993, p.169-212.

${ }^{2}$ FINDENEGG, I. Factors controlling primary productivity especially with regard to water replenishment, stratification, and mixing. In: GOLDMAN, C. R. (Ed.). Primary Productivity in Aquatic Environments. Berkeley: University of California Press, 1974, p. 107-119.
} 
Os organismos aquáticos apresentam estruturas morfológicas e fisiológicas que permitem sua sobrevivência no ambiente, estabelecendo-se condições criadas pela eutrofização e outras adaptações fisiológicas observadas. Desse modo, sobrevivem organismos adaptados às novas condições estabelecidas (BRANCO, 1986). Portanto, para a avaliação de um corpo d'água, mostra-se relevante o estudo da comunidade aquática e das características físicas e químicas do ambiente que interagem com os organismos, determinando os agentes causadores das alterações e as consequiências geradas para o ecossistema (GENTIL, 2000).

Dentre as populações de organismos planctônicos (que flutuam na coluna d’água, sem ou com algum movimento próprio), a que mais se destaca na classificação ecológica dos corpos d'água é a do fitoplâncton, que inclui as algas e as Cyanobacteria, justamente pela sua presença em todo tipo de água continental, além de incluir espécies tolerantes à presença de poluentes, que respondem prontamente às mudanças ocorridas. Desse modo, registram melhor as variações da qualidade da água, funcionando como indicadores biológicos e permitindo o registro de alterações presentes e passadas, assim como o desequilíbrio existente (GENTIL, 2000).

As Cyanobacteria são organismos que não possuem membrana nuclear (procariontes), porém, com um sistema fotossintetizante oxigênico semelhante ao das algas (vegetais), sendo, portanto, produtores primários. São também conhecidas pela dupla denominação de cianofíceas (ou algas azuis) e de Cyanobacteria.

De acordo com Calijuri (1999), alterações na composição e abundância da comunidade fitoplanctônica podem ser provocadas pela alteração ambiental que, ocorrendo com frequiência e intensidade variáveis, pode apresentar-se como perturbação que irá então modificar o caráter qualitativo e quantitativo da biota, selecionando espécies através de mecanismos competitivos, que se tornam então favorecidas.

Segundo Reynolds (1984) ${ }^{3}$ apud (TUCCI, 2002), a comunidade fitoplanctônica pode apresentar ciclos sazonais e anuais relacionados com as variações ambientais das diferentes épocas do ano. Margalef (1983) também se refere aos organismos planctônicos como "sensores" das variações ambientais, pois respondem rapidamente às alterações ambientais através de sua composição e abundância. Dessa forma, acompanhar as variações na estrutura da comunidade ao longo de um ciclo sazonal reveste-se de grande importância, quando se

\footnotetext{
${ }^{3}$ REYNOLDS, C.S. Phytoplankton periodicity: the interations of form, function and environmental variability. Freshwater Biology, v. 14, p. 111-142, 1984.
} 
pretende entender o funcionamento do ecossistema tanto para o monitoramento quanto para a recuperação de ambientes aquáticos (TUCCI, 2002).

O aporte de nutrientes em corpos hídricos, advindos de efluentes domésticos e industriais, fertilizantes agrícolas e de outras fontes, leva à eutrofização das águas propiciando, assim, condições ideais para a proliferação do fitoplâncton. Elevadas concentrações de nutrientes na água, principalmente fosfato e nitrogênio, aumento da intensidade luminosa e da temperatura da água (de 15 a $30{ }^{\circ} \mathrm{C}$ ), $\mathrm{pH}$ com valores entre 6 e 9 e altas taxas de evaporação durante os períodos de estiagem contribuem, significativamente, para a proliferação excessiva do fitoplanctôn, o que é denominado floração (ZAGATTO et al., 1997). Dentre esses organismos destacam-se as Cyanobacteria, que atraem a atenção não só de pesquisadores e técnicos que controlam a qualidade das águas, mas também do público em geral, por sua resposta imediata à eutrofização de reservatórios, sob forma de florações.

As principais conseqüências do desenvolvimento e posterior degradação das florações de Cyanobacteria são a desoxigenação das águas, alteração das características organolépticas da água e produção de toxinas por algumas espécies (VASCONCELOS e ARAÚJO, 1994).

Segundo Zagatto et al. (1997), florações de Cyanobacteria dos gêneros Microcystis, Oscillatoria, Anabaena, Aphanizomenon, Nodularia, Trichodesmium e Cylindrospermopsis, têm causado diversos problemas à saúde humana. Cabe ressaltar que uma mesma espécie pode apresentar linhagens tóxicas ou não, sendo que essas variações se devem a fatores genéticos e/ou ambientais.

De acordo com Vasconcelos e Araújo (1994), as toxinas produzidas pelas Cyanobacteria, ou cianotoxinas, são essencialmente de três tipos, de acordo com os efeitos: neurotoxinas, hepatotoxinas e irritantes ao contato. A gravidade da intoxicação depende da dose ingerida ou da densidade dos organismos no corpo d’água.

As neurotoxinas agem no sistema nervoso, causando bloqueio neuromuscular que leva à morte do organismo afetado por parada respiratória em poucos minutos (CHORUS e BARTRAM, 1999; ZAGATTO et al., 1997). As hepatotoxinas agem no fígado e a ingestão contínua de baixas doses pode ter como conseqüência alterações citoquímicas crônicas das células hepáticas, contribuindo para o desenvolvimento de tumores (VASCONCELOS e ARAÚJO, 1994). Assim como todas as células Gram-negativas, as Cyanobacteria apresentam lipopolissacarídeos (LPSs) como componentes de membrana externa da parede, que podem produzir respostas como irritação ou alergias em pessoas ou animais que entrem em contato com os compostos da água (CHORUS e BARTRAM, 1999). 
O primeiro relato de intoxicação de animais causada por floração de Cyanobacteria é de 1878, no lago Alexandrina, na Austrália, onde morreram ovelhas, cavalos e cães (BRANCO, 1959). Desde então, inúmeros casos de intoxicação foram descritos em diversas partes do planeta, como em 1930, nos Estados Unidos, onde cerca de 10000 pessoas apresentaram distúrbios gastrointestinais, ao consumirem água de manancial com floração. Em 1975, na Pensilvânia, EUA, cerca de 5000 pessoas tiveram o mesmo sintoma após ingestão de água com floração de Cyanobacteria. Na Austrália, cerca de 150 pessoas foram hospitalizadas com hepatoenterite, devido ao consumo de água com toxina de Cylindrospermopsis raciborskii, a qual fora tratada com o algicida sulfato de cobre. Este produto causa a lise das células das Cyanobacteria, liberando as toxinas para a água (ZAGATTO et al., 1997). As toxinas não são totalmente removidas pelos tratamentos usuais de água, podendo manter-se na água de consumo (VASCONCELOS e ARAÚJO, 1994).

No Brasil, alguns casos de mortandade de animais domésticos e intoxicação humana foram relacionados às florações (BEYRUTH et al., 1992). Em 1996, mais de 60 pessoas morreram devido à utilização de água contaminada por hepatotoxina produzida por Cyanobacteria, em uma clínica de hemodiálise em Caruaru, no Estado de Pernambuco (CARMICHAEL, 1998). Neste caso, a água não recebeu tratamento completo mantendo ainda células em suspensão, causando a sua lise com consequente liberação das toxinas para a água (AZEVEDO, 1998).

Além das Cyanobacteria, outros organismos do fitoplâncton constituem o primeiro elo da cadeia alimentar planctônica e, ao lado das macrófitas aquáticas, iniciam a fase biológica do ciclo dos nutrientes nos ambientes aquáticos, permitindo seu ingresso na cadeia alimentar. Produzem parte considerável do oxigênio atmosférico e oxigênio dissolvido na água, essencial aos organismos aeróbios, além de prover alimento para os consumidores primários incluindo protozoários, insetos, organismos do zooplâncton e peixes, servindo ainda aos organismos da cadeia detritívora tais como vírus, bactérias e fungos, tanto de substrato como de alimento. Dois terços da superfície terrestre são cobertos por água, e como o fitoplâncton é a comunidade das águas superficiais, torna-se responsável pela produção primária na maior parte da superfície terrestre (BEYRUTH, 1996).

A produtividade primária nos ambientes aquáticos reflete imediatamente o grau de eutrofização da água, resultante da contribuição dos tributários e do uso e ocupação das bacias de captação, pois ao lado dos organismos da cadeia detritívora, o fitoplâncton e as macrófitas livre-flutuantes são os principais organismos a assimilarem nutrientes e contaminantes da 
água. O fitoplâncton é o produtor primário mais significativo nos ambientes em que predomina a água aberta, ou seja, sem a influência das margens (BEYRUTH, 1996).

Segundo Rocha ${ }^{4}$ (1992) apud Beyruth (1996), cada espécie do fitoplâncton está relacionada a outras espécies e requer diferentes características estruturais físicas, químicas e biológicas intrínsecas ao seu hábitat.

Para que o fitoplâncton se mantivesse na massa de água seria necessário que sua densidade fosse igual a um. No entanto, a maioria das espécies fitoplanctônicas tem densidade superior à da água (1,01 a 1,05, podendo haver espécies com valores superiores a 2). Várias adaptações possibilitaram a esses organismos superar as desvantagens de sua alta densidade. As principais são as seguintes: formação de bainha mucilaginosa, cuja densidade é próxima à da água, é uma das adaptações freqüentes em muitas espécies de clorofíceas e Cyanobacteria; gotículas de óleo, através das quais os organismos podem aumentar sua capacidade de flutuação, podendo ser encontradas em algumas espécies de clorofíceas, fitoflagelados e diatomáceas (ESTEVES, 1998). Em várias espécies de Cyanobacteria ocorre a presença de aerótopos (vacúolos gasosos) para facilitar sua flutuação na coluna d'água.

A presença de aerótopos em muitas espécies de Cyanobacteria permite que as células regulem sua flutuação em resposta à disponibilidade de luz e nutrientes (KLEMER e KONOPKA, 1989). Este mecanismo promove a manutenção das Cyanobacteria na zona eufótica de forma muito mais eficiente e vantajosa que outros grupos fitoplanctônicos. Além disso, controlando o aumento ou diminuição do número de aerótopos nas células, têm capacidade de regular sua posição na coluna d'água para melhor se adaptarem à intensidade luminosa (KLEMER, 1991).

Uma das maiores dificuldades na utilização do fitoplâncton como indicador de poluição é sua identificação em nível de espécie. Em estudos sanitários o fitoplâncton é de importância fundamental como indicativo da qualidade da água e possibilita a realização de previsões a partir de alterações ambientais. Se o estudo for realizado com base em espécie, a qualidade da informação adquirida amplia-se consideravelmente, pois as espécies fitoplanctônicas podem servir para indicar desde a qualidade do produto água para abastecimento até o grau de dificuldade apresentado para o tratamento da água bruta, o potencial de produção de toxinas e a possibilidade de manejo através da biomanipulação,

\footnotetext{
${ }^{4}$ ROCHA, A.A. Algae as biological indicators of water pollution. In: CORDEIRO-MARINO, M. et al. (Ed.). Algae and environmental: a general approch. São Paulo: Sociedade Brasileira de Ficologia, 1992, p.34-52.
} 
utilizando o fitoplâncton para manutenção de um nível satisfatório de qualidade (BEYRUTH, 1996).

Particularmente sobre os reservatórios em estudo, existem poucos trabalhos em relação à ecologia da comunidade fitoplânctônica. De acordo com Souza et al. (1998), o Reservatório Billings foi estudado durante um ano, com objetivo de analisar a qualidade ambiental. Vários parâmetros físicos, químicos e biológicos foram avaliados. Amostras do fitoplâncton foram coletadas mensalmente em oito estações, incluindo o braço do Rio Pequeno. Um total de 69 táxons foi registrado, ocorrendo dominância de Cyanobacteria.

Carvalho (2003) avaliou a composição fitoplanctônica de seis reservatórios de abastecimento público no Estado de São Paulo para selecionar indicadores e índices biológicos. Dentre estes, o Reservatório Billings apresentou 154 táxons e as Cyanobacteria foram dominantes em todo período de estudo.

Zagatto (1995) realizou um trabalho de avaliação ecotoxicológica no Reservatório Guarapiranga, constatando mortandade de animais em decorrência das florações de Cyanobacteria tóxicas.

Segundo Beyruth (1996), estudos da comunidade fitoplanctônica, bem como dos aspectos ecológicos e sanitários no Reservatório Guarapiranga, confirmaram as florações de Cyanobacteria decorrentes do aporte de nutrientes nesse reservatório.

Matsuzaki (2007) avaliou os aspectos sanitários das águas do Reservatório Guarapiranga após a transposição das águas do braço Taquacetuba do Reservatório Billings, constatando alto índice de eutrofização desses reservatórios, assim como intensa dominância das Cyanobacteria nesses ambientes.

Além desses trabalhos, não há na literatura brasileira outros dados sobre ecologia da comunidade fitoplanctônica em reservatórios de abastecimento público. A maioria dos trabalhos de ecologia do fitoplâncton discute os resultados em nível de classes, não se tendo muitas informações sobre as espécies (SANT’ANNA e AZEVEDO, 2000).

A decisão de recuperar ou preservar bacias hidrográficas destinadas ou não ao abastecimento público pode implicar em altos custos, envolvendo profundas modificações em situações já estabelecidas e a necessidade de impedir distorções quanto ao uso e ocupação do solo. Entretanto, o custo operacional necessário ao tratamento da água de reservatórios eutrofizados pode ser muito superior ao custo da reabilitação ou recuperação da qualidade da água dos reservatórios já existentes (BEYRUTH, 1996). 


\section{CONCLUSÕES}

- Os resultados mostraram que os principais fatores ambientais que interferiram na dinâmica da comunidade fitoplanctônica foram: elevados valores de temperatura da água, de $\mathrm{pH}$ e de nutrientes.

- As Classes Chlorophyceae e Cyanobacteria tiveram altas densidades em ambos os reservatórios, independentemente da época do ano, comprovando em parte a primeira hipótese do estudo, pois em termos qualitativo os dois reservatórios se mostraram semelhantes, porém quantitativamente no Reservatório Billings, as Cyanobacteria foram dominantes e abundantes durante todo período de estudo.

- Bacillariophyceae foram abundantes em abril/2002 no Reservatório Billings. No Reservatório Guarapiranga, Zygnemaphyceae foram abundantes no inverno e primavera. As demais classes: Chrysophyceae, Cryptophyceae, Dinophyceae, Euglenophyceae e Xanthophyceae foram raras em ambos os reservatórios.

- Espécies potencialmente tóxicas (Cylindrospermopsis raciborskii, Microcystis panniformis, M. protocystis, M. wesenbergii, Planktothrix agardhii) de Cyanobacteria foram detectadas no Reservatório Guarapiranga, onde antes eram ausentes, comprovando a segunda hipótese do estudo. No Reservatório Billings, as Cyanobacteria ocorreram durante todo o período de estudo.

- As espécies descritoras e os grupos funcionais foram representados, respectivamente, por Anabaena circinalis (H1), Microcystis aeruginosa (LM/M) e Mougeotia sp. (T) no Reservatório Guarapiranga e C. raciborskii $\left(\mathbf{S}_{\mathrm{N}}\right), M$. aeruginosa e $M$. panniformis (LM/M), $P$. agardhii e $P$. cf. clathrata (S1) no Reservatório Billings.

- A Portaria no 518 de 25 de março de 2004 do Ministério da Saúde (BRASIL, 2004), estabelece o monitoramento de Cyanobacteria e cianotoxinas pelos órgãos responsáveis pela operação de sistema de abastecimento de água. O presente estudo demonstrou a grande importância do fitoplâncton, descrevendo as espécies descritoras 
como parte desse monitoramento, sugerindo novos estudos para posterior aprimoramento do estudo dessas e de outras espécies que possam ocorrer nos reservatórios de abastecimento público. 


\section{REFERÊNCIAS}

AMERICAN PUBLIC HEALTH ASSOCIATION. Standard methods for the examination of water and wastewater. $18^{\text {th }}$ ed. Washington, D.C.: APHA, 1998. p. 1-1268.

AZEVEDO, S. M. F. O. Toxinas de Cianobactérias: Causas e conseqüências para a Saúde Pública. Revista Virtual de Medicina, v. 1, n. 3-A.1, 1998. Disponível em: <www.medonline.com.br/med_ed/med3/microcis.htm>. Acesso em: 16 jun. 2002.

BEYRUTH, Z. Comunidades fitoplanctônica da Represa de Guarapiranga: 1991-92. Aspectos ecolocógicos, sanitários e subsídios para reabilitação da qualidade ambiental. Tese (Doutorado) - Faculdade de Saúde Pública da Universidade de São Paulo, São Paulo, 1996.

BEYRUTH, Z. Water quality of Guarapiranga Reservoir, São Paulo, Brazil, 1991-1992. Verhandlungen Internationalen Vereinigung für theoretische ünd angeivanate Limnologie, v. 26, p. 675-683, 1997.

BEYRUTH, Z. Periodic disturbances, trophic gradient and phytoplankton characteristics related to cyanobacterial growth in Guarapiranga Reservoir, São Paulo State, Brazil. Hydrobiologia, v. 424, p. 51-65, 2000.

BEYRUTH, Z.; SANT'ANNA, C. L.; AZEVEDO, M. T. P.; CARVALHO, M. C.; PEREIRA, H. A. S. L. Toxic Algae in Freshwaters of São Paulo State. Algae and Environment: A general Approch. São Paulo: SBFic/CETESB, 1992.

BICUDO, C. E. M.; BICUDO, D. C.; CASTRO, A. A. J.; PICELI-VICENTIM, M. M. Fitoplâncton do trecho a represar do Rio Paranapanema (Usina Hidrelétrica de Rosana), estado de São Paulo, Brasil. Revista Brasileira de Biologia, v. 52, n. 2, p. 293-310, 1991.

BOUVY, M.; MOLICA, R.; DE OLIVEIRA, S.; MARINHO, M.; BEKER, B. Dynamics of a toxic cyanobacterial bloom (Cylindrospermopsis raciborskii) in a shallow reservoir in the semi-arid region of northeast Brazil. Aquatic Microbial Ecology, v. 20, p. 285-297, 1999. 
BRANCO, S. M. Algas tóxicas: controle das toxinas em águas de abastecimento. Revista DAE, v. 20 , n. 35 , p. 47-53, 1959.

BRANCO, S. M. Hidrobiologia aplicada à engenharia sanitária. 3 ed. São Paulo: CETESB/ASCETESB, 1986.

BRASIL. Ministério da Saúde. Secretaria de Vigilância Sanitária. Portaria no 518, de 3 de março de 2004. Estabelece os procedimentos e responsabilidades relativos ao controle e vigilância da qualidade da água para consumo humano e seu padrão de potabilidade. Brasília, DF: Ministério da Sáude, 2004.

CALIJURI, M. C. A comunidade fitoplanctônica em um reservatório tropical (Barra Bonita, SP). Tese (Livre docência) - Escola de Engenharia de São Carlos, Universidade de São Paulo, São Carlos, 1999.

CAPOBIANCO, J. P. R.; WHATELY, M. Billings 2000: ameaças e perspectives para o maior reservatório de água da região metropolitana da bacia hidrográfica da Billings no período 1989-99. São Paulo: Instituto Socioambiental, 2002. p. 60.

CARLSON, R. E. A Trophic State Index for lakes. Limnology and Oceanography, v. 22, p. 361-369, 1977.

CARMICHAEL, W. W. Microcystin concentrations in human livers, estimation of human lethal dose - lessons from Caruaru, Brazil. In: INTERNATIONAL CONFERENCE ON TOXIC CYANOBACTERIA, 4., Beaufort. Abstract... Beaufort, Department of Pharmacology, University of Adelaide, 1998. p. 115. Seção 4.

CARVALHO, M. C. Comunidades fitoplanctônica como instrumento de biomonitoramento de reservatórios no Estado de São Paulo. Tese (Doutorado) Departamento de Saúde Ambiental da Faculdade de Saúde Pública da Universidade de São Paulo, São Paulo, 2003.

CHORUS, I.; BARTRAM, J. Toxic Cyanobacteria in Water. Londres: E \& FN Spon, 1999. p. 416.

COLE, G. A. Textbook of Limnology. Saint-Louis, MO, USA: The C. V. Mosby, 1975. p. 283. 
DELLAMANO-OLIVEIRA, M. J. Comunidade fitoplanctônica do reservatório de barra Bonita e sua relação com a composição e quantidade de polissacarídeos estracelulares e agregados gelatinosos. Tese (Doutorado) - Departamento de Ecologia e Recursos Naturais da Univesidade Federal de São Carlos, São Carlos, 2006.

DEPARTAMENTO DE ÁGUAS E ENERGIA ELÉTRICA. Sistema Integrado de Gerenciamento de Recursos Hídricos do Estado de São Paulo, 2001. Disponível em: <www.sigrh.sp.gov.br/bancodedados/plu.htm>. Acesso em: 06 maio 2005.

ESTEVES, F. A. Fundamentos de Limnologia. 2. ed. Rio de Janeiro: Interciências, 1998.

GENTIL, R. C. Variação sazonal do fitoplâncton de um lago subtropical eutrófico e aspectos sanitários, São Paulo, SP. Dissertação (Mestrado) - Faculdade de Saúde Pública da Universidade de São Paulo, São Paulo, 2000.

GENTIL, R. C. Estrutura da comunidade fitoplanctônica de pesqueiros da Região Metropolitana de São Paulo, SP, em dois períodos: primavera e verão. Tese (Doutorado) - Instituto de Botânica. Secretaria de Meio Ambiente do Estado de São Paulo, São Paulo, 2007.

HASLE, G.R.; FRYXELL, G. A. Diatoms: cleaning and mouting for light and electron microscopy. Transactions of the American Microscopical Society, v. 89. p. 469-474, 1970.

HUSZAR, V. L. M.. Planktonic algae, other than desmids, of three Amazonian systems (Lake Batata, Lake Mussurá and Trombetas River), Pará, Brazil. Amazoniana. v. 14. n. 1/2, p. 37 73, 1996.

HUSZAR, V. L. M.; SILVA, L. H.; MARINHO, M.; DOMINGOS, P.; SANT'ANNA, C.L Cyanoprokaryote assemblages in eight productive tropical Brazilian waters. Hydrobiologia, v. 424, p. 67-77, 2000.

INSTITUTO DE ENGENHARIA. Seminário: “O abastecimento de água na macro-região de São Paulo: perspectivas a curto, médio e longo prazo" realizado nos dias 20 e 21 novembro/2003, Fusp. Disponível em: <www.ie.org.br>. Acesso em: 20 jan. 2004.

KLEMER, A. R. Effects of nutritional status on cyanobacteria buoyancy,bloom, and dominance, with special reference to inorganic carbon. Canadian Journal of Botany, v. 69 , p. $1133-1138,1991$. 
KLEMER, A. R.; KONOPKA, A.E. Causes and consequences of blue-green algal (cyanobacterial) bloom. Lake and Reservoir Management, v. 5, n. 1, p. 9-19, 1989.

LAMPARELli, M. C. Graus de trofia em corpos d'água do Estado de São Paulo: Avaliação dos métodos de monitoramento. Tese (Doutorado) - Instituto de Biociências da Universidade de São Paulo, São Paulo, São Paulo, 2004.

LLOYD, M.; GHELARDI, R. J. A table for calculating the equitability component of species diversity. Journal of Animal Ecology, v. 33, p. 217-225, 1964.

LOBO, E.; LEIGHTON, G. Estruturas comunitárias de lãs fitocenosis planctônicas de los sistemas de desembocaduras de rios y esteros de la zona central de Chile. Revista de Biologia Marinha, v. 22, n. 1, p. 1-29, 1986.

LUND, J. W. G.; KIPLING, C.; LECREN, E. D. The inverted microscope method of estimating algal number and the statistical basis of estimating by counting. Hydrobiologia, v. 11, p. 143-170, 1958.

MARGALEF, R. Limnología. Barcelona: Ed. Omega, 1983.

MATSUZAKI, M. A comunidade fitoplânctônica de um pesqueiro na cidade de São Paulo: Aspectos ecológicos e sanitários. Dissertação (Mestrado) - Departamento de Saúde Ambiental da Faculdade de Saúde Pública da Universidade de São Paulo, São Paulo, 2002.

MATSUZAKI, M. Transposição das águas do braço Taquacetuba da represa Billings para a represa Guarapiranga: aspectos relacionados à qualidade de água para abastecimento. Tese (Doutorado) - Departamento de Saúde Ambiental da Faculdade de Saúde Pública da Universidade de São Paulo, São Paulo, 2007.

MATSUZAKI, M.; MUCCI, J. L. N.; ROCHA, A. A. Phytoplankton community in a recreational fishing lake, Brazil. Revista de Saúde Pública, v. 38, n.5, p. 679-686, 2004.

ODUM, E. P. Ecologia. Rio de Janeiro: Ed. Guanabara, 1988.

PADISÁK, J. Cylindrospermopsis raciborskiiI (Woloszynska) Seenayya et Subba Raju, an espanding, highly adaptive cyanobacterium: worldwide distribution and review of its ecology. Archiv Hydrobiologia, v. 107. n. 4. p. 563-593, 1997.

PRESOTTO, A.; CABIANCA, M. A. A. Pesqueiros sob uma visão integrada de meio ambiente, saúde pública e manejo: um estudo na Região Metropolitana de São Paulo. In 
ESTEVES, K. E.; SANT'ANNA, C. L. (Org.). Caracterização das Áreas de Localização de 30 Pesqueiros da Região Metropolitana de São Paulo. São Carlos: Rima, 2006. p. 19-36.

SANT'ANNA, C. L.; AZEVEDO, M. T. P. Contribution to the knowledge of potentially toxic Cyanobacteria from Brazil. Nova Hedwigia, v. 71, p. 359-385, 2000.

SANT'ANNA, C. L.; AZEVEDO, M. T. P.; SORMUS, L.. Fitoplâncton do Lago das Garças, Parque Estadual das Fontes do Ipiranga, São Paulo, SP, Brasil: Estudo Taxonômico e Aspectos Ecológicos. Hoehnea, v. 16, p. 89-131, 1989.

SANT'ANNA, C. L.; SORMUS, L.; TUCCI, A.; AZEVEDO, M. T. P. Variação sazonal do fitoplâncton do Lago das Garças, São Paulo, SP Brasil. Hoehnea, v. 24, p. 67-86, 1997.

SANT'ANNA, C. L.; AZEVEDO, M. T. P.; AGUJARO, L. F.; CARVALHO, M. C.; CARVALHO, L. R.; SOUZA, R. C. R. Manual Ilustrado para Identificação e Contagem de Cianobactérias Planctônicas de Águas Continentais Brasileiras. Rio de Janeiro: Interciência, 2006.

SANT'ANNA, C. L.; MELCHER, S. S.; CARVALHO, M. C.; GEMELGO, M. C. P.; AZEVEDO, M. T. P. Planktic Cyanobacteria from upper Tietê basin reservoirs, SP, Brazil. Revista Brasileira de Botânica, v. 30, p. 1-17, 2007.

SÃO PAULO (SP). Secretaria do Meio Ambiente e Coordenadoria de Educação Ambiental. Centro de Estudos de Cultura Contemporânea. Consumo, lixo e meio ambiente: desafios e alternativas. São Paulo: CEDEC, 1997.

SÃO PAULO (SP). Companhia de Tecnologia de Saneamento Ambiental. Determinação de pigmentos fotossintetizantes: clorofila-a, b e c e feofitina-a: Método de ensaio. Norma Técnica L5.306. São Paulo: CETESB, 1990. p. 1-19.

SÃO PAULO (SP). Secretaria do Meio Ambiente. Companhia de Tecnologia de Saneamento Ambiental. Relatório de Qualidade das Águas Interiores do Estado de São Paulo. São Paulo: CETESB, 1997. p. 289. (Série Relatórios).

SÃO PAULO (SP). Secretaria do Meio Ambiente. Companhia de Tecnologia de Saneamento Ambiental. Relatório de Qualidade das Águas Interiores do Estado de São Paulo. São Paulo: CETESB, 2004. p. 273. (Série Relatórios). 
SÃO PAULO (SP). Secretaria do Meio Ambiente. Companhia de Tecnologia de Saneamento Ambiental. Relatório de Qualidade das Águas Interiores do Estado de São Paulo. São Paulo: CETESB, 2007. p. 327. (Série Relatórios).

SHANNON, C.E.; WEAVER, W. The mathematical theory of communication. Urnaba, Illinois: University Press, 1963. p. 177.

SILVA, L. H. S. Fitoplâncton de um reservatório eutrófico (Lago Monte Alegre), Ribeirão Preto, São Paulo. Revista Brasileira de Biologia, v. 59. n.2, p. 281-303, 1999.

SIMPSON, E. H. Measurement of diversity. Nature, p. 163-688, 1949.

SOUZA, R. C. R.; CARVALHO, M. C.; TRUZZI, A. C. Cylindrospermopsis raciborskii (Wolosz.) Seenaya and Subba Raju (Cyanophyceae) dominance and a contribution to the knowledge of Rio Pequeno arm, Billings Reservoir, Brazil. Environmental Toxicology and Water Quality, v. 13, p. 73-81, 1998.

STRAŠBRABA, M.; TUNDISI, J. G. Gerenciamento da qualidade da água de represas. V. 1. In TUNDISI, J. G. (Ed.). Diretrizes para o gerenciamento de Lagos. São Carlos: ILEC, IIE, 2000, p. 280.

TUCCI, A. Sucessão da comunidade fitoplanctônica de um reservatório urbano e eutrófico, São Paulo, SP, Brasil. Tese (Doutorado) - Instituto de Biociências da Universidade Estadual Paulista, Rio Claro, 2002.

TUCCI, A.; SANT'ANNA, C. L. Cylindrospermopsis raciborskii (Wloszynska) Seenayya and Subba Raju (Cyanobacteria): variação semanal e relações com fatores ambientais em um reservatório eutrófico, São Paulo, SP, Brasil. Revista Brasileira de Botânica, v. 26, p. 97 $112,2003$.

TUNDISI, J. G. Água no Século XXI: enfrentando a escassez. São Carlos: Rima/IIE, 2003. v. 1 .

VASCONCELOS, V.; ARAÚJO, F. Cianobactérias: Um risco para o Ambiente e para a Saúde Humana. Porto - Portugal: Ministério da Saúde, 1994.

VERCELLINO, I. S. Respostas do perifíton aos pulsos de enriquecimento em níveis crescentes de fósforo e nitrogênio em represa tropical mesotrófica (Lago das Ninféias, São Paulo). Tese (Doutorado) - Instituto de Biociências da Universidade Estadual Paulista, Rio Claro, 2007. 
XAVIER, M. B. Fitoplâncton do Rio Grande, Represa Billings, São Paulo, Brasil: Estudo Taxonômico (1985-1986). IHERINGIA, Sér. Bot., v. 47, p. 103-122, 1996.

ZAGATTO, P. A. Evaluation ecotoxicologique du reservoir Guarapiranga, SP-Brésil, en relation avec le probléme des algues toxiques et des algicides. $\mathrm{Ph}$. D. Thesis - Université de Metz, Centre dês Sciences de I'Environement, France, 1995.

ZAGATTO, P. A.; ARAGÃO, M. A.; CARVAlHO, M. C.; SOUZA, R. C. R. Manual de Orientação em Casos de Florações de Algas Tóxicas: Um problema Ambiental e de Saúde Pública. São Paulo: CETESB, 1997. 